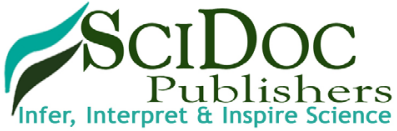

\section{Fibrin Glue Mesh Fixation (GMF) Vs Sutures Mesh Fixation (SMF) In Human Open Inguinal Hernia Repair (OIHR): Surgically and Histologically}

Ghonimi WAM ${ }^{1 *}$, Elhorbity MA²

${ }^{1}$ Department of Histology and Cytology, Faculty of Veterinary Medicine, Zagazig University, Zagazig, Egypt.

${ }^{2}$ Department of General Surgery, Benha Teaching Hospital, General Organization for Teaching Hospitals and Institutes, Benha, Egypt.

\title{
Abstract
}

Background: The Lichtenstein technique; suture mesh fixation is widely used for the open inguinal hernia repair (OIHR) as it is easy to learn and is associated with a low rate of complications and recurrences. However, recently, some studies have clarified that this technique is inferior to some 'sutureless' repair systems in terms of perceived difficulty, operating time, postoperative pain, patient satisfaction, etc.

Methods: We employed a fibrin glue mesh fixation in 30 patients (group A) and sutures mesh fixation using vicryl in 30 patients (group B). All the patients included in this study were male and aged between 17 and 60 years old with exclusion of recurrent, obstructed and strangulated hernia. Histologically, at the end of the Fibrin Glue Mesh Fixation, small pieces from the mesh fixed with Posterior wall of the inguinal canal; conjoint tendon were taken for the histological studies. The specimens were fixed immediately in neutral buffered formalin $10 \%$, dehydrated, cleared and processed for paraffin blocks and 5 micron thick sections were obtained, mounted on a glass slides and stained histologically with Hematoxylin \& Eosin (H\&E) and Green Masson's Trichrome.

Results: The mean operating time was ranged from 30 to 45 minutes in a fibrin glue mesh fixation (group A) and from 40 to 60 minutes in a suture mesh fixation (group B). All patients received spinal anesthesia and discharged home $24 \mathrm{~h}$ after the operation and none required readmission to hospital. There were no intra operative complications. No sepsis, mesh rejection or other complications such as haematoma or seroma were recorded at 14-day follow-up. Regarding the postoperative pain, patients of group A were returned to their normal activity from 10 days to 2 weeks after operation with better satisfaction. Meanwhile, patients of group B take more time from 2 weeks to 6 weeks for returning to normal activity with less satisfaction. Histologically, with H\&E and Green Masson's Trichrome stains, colorless spherical structures of the posterior wall of the inguinal canal-conjoint tendon were observed. Meanwhile, under polarized light, the brightly birefringent were observed filling these colorless structures. Furthermore, with Green Masson's Trichrome stain, massive wavy fibrin threads forming bundles were observed filling all the spaces in between the polypropylene threads of mesh fixing the later with the surrounding tissues. Moreover, numerous fibroblasts were observed in between the fibrin bundles which distributed in different directions.

Conclusion: The present investigation clarify that the use of fibrin glue is better than sutures in tension free inguinal hernia repair using mesh technique. Moreover, there is less operative time, post-operative pain, with better patient satisfaction and early return to normal activities in group A using fibrin glue mesh fixation in inguinal hernia repair.

Keywords: Fibrin Sealant; Thrombin; Glue; Inguinal Hernia; Suture Mesh Fixation; Glue Mesh Fixation.

\section{Introduction}

Recently, the trauma experienced by patients undergoing inguinal hernia repair has been reduced due to advances in surgical techniques. Of the current surgical approaches, the Lichtenstein technique is widely used because it is easy to learn and is associated with a low rate of complications and recurrences $[1$, 2]. To further progress this field, recent studies have focused on two issues (1) the incidence of groin pain at long-term follow-up $[3,4]$; and (2) the teaching issues that determine the progress of

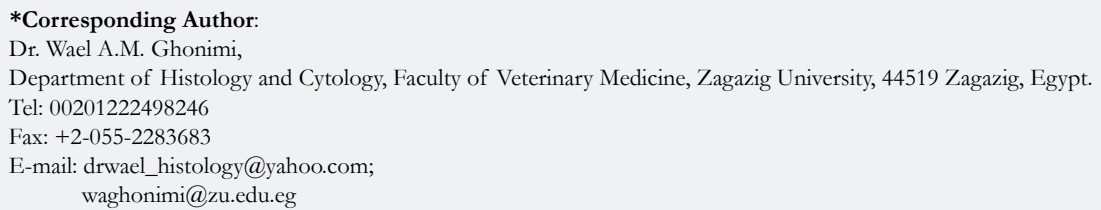

Citation: Ghonimi WAM, Elhorbity MA (2017) Fibrin Glue Mesh Fixation (GMF) Vs Sutures Mesh Fixation (SMF) In Human Open Inguinal Hernia Repair (OIHR): Surgically and Histologically. Int J Surg Res. 4(3), 75-81. doi: http://dx.doi.org/10.19070/2379-156X-1700016 and reproduction in any medium, provided the original author and source are credited. 
surgeons training in this technique [5]. For example, Nienhuijs et al., reported that the Lichtenstein technique is inferior to the Prolene Hernia System (Ethicon Inc., Somerville, NJ, USA) and to the mesh plug repair system in terms of operating time, incision length, perceived difficulty and surgeon satisfaction [5]. It has been hypothesised that these results are linked to the use of sutures for mesh fixation [6].

Fibrin sealant or glue is a unique surgical hemostatic/sealant/ adhesive material that is being used with increasing frequency in a variety of surgical situations. In practice, there is a twocomponent system in which a solution of concentrated fibrinogen and factor XIII are combined with a solution of thrombin and calcium to form a coagulum that simulating the final stage of the clotting cascade. Once the thrombin/calcium is combined with the fibrinogen/factor XIII, a fibrin clot forms in seconds, or somewhat slower if a more dilute form of thrombin is used. In some preparations, or in selected indications, an antifibrinolytic agent is included to prevent lysis of the clot $[7,8]$. Antifibrinolytic agents such as aprotinin are included in the preparation to enhance the life span of the sealant and prolong its effectiveness. In addition to the haemostatic action of the preparation, the fibrinogen component gives the product its tensile strength and adhesive properties, and the thrombin component promotes fibroblast proliferation [9]. Furthermore, thrombin is an enzyme that converting fibrinogen to fibrin monomers within 10 to 60 seconds, giving rises to a three-dimensional gel [10].

The search for the perfect operative glue began in the first decade of the $20^{\text {th }}$ century. By the 1940 s, fibrinogen and thrombin were combined in operative settings. When Cohn fractionation led to the ability to generate highly concentrated fibrinogen preparations (in the 1960s), fibrin glues were used to promote wound healing, skin grafting and dural sealing, to provide hemostasis in microvascular surgery and parenchymal injury, to serve as a matrix for bony chips and fragments in the repair of bone defects [11].

The aim of current investigation is to assess the advantages of fibrin glue over sutures (vicryl) in fixation of the mesh in open inguinal hernia repair.

\section{Subjects and Methods}

Between January 2015 and May 2016, a total of 60 patients, with inguinal hernia, by Linchtenstein free tension hernia repair using mesh technique were done. The mesh used in both fibrin glue and suture mesh fixation is $6 \times 11 \mathrm{~cm}$ non-absorbable polypropylene mesh. All patients were informed about the purpose of the study with ethical aspects and a written consent was taken.

All patients were randomized into 2 groups. Group A (fibrin glue mesh fixation in 30 patients) and group B (sutures mesh fixation using vicryl 2-0 in 30 patients). All the patients included in this study were male and aged between 17 and 60 years old with exclusion of recurrent, obstructed and strangulated hernia. Also patients with known hypersentivity to any drug medication or blood product sensitivity were excluded.

In group A the mesh was fixed to the posterior wall of the inguinal canal (conjoint tendon and inguinal ligament) after excision of the hernia sac by using fibrin glue without use any sutures (one kit for each case) each kit composed of 2 vial.

In group $\mathrm{B}$, the mesh was fixed to the posterior wall of the inguinal canal after excision of the hernia sac by using multiple stitches (vicryl 2-0).

Duration of surgery, early and late post-operative pain, duration of hospital stay and return to normal activities and work were recorded in both groups.

All patients in both groups received spinal anesthesia. All patients were prescribed $75 \mathrm{mg}$ diclofenac intramuscular (I/M) each 12 hour in the early 24 hour postoperative and additional dose of $50 \mathrm{mg}$ tramadol intravenous (I/V) slowly when needed. After hospital discharge all patient were prescribed oral $75 \mathrm{mg}$ diclofenac each 12 hour in the early post-operative days or when needed and additional dose of $100 \mathrm{mg}$ tramadol intra muscular when needed. All patients were informed to ask for analgesia if the prescribed post-operative analgesia not effective. The primary end points of each group were severity of pain, analgesia requirement and incidence of haematoma or theroma formation.

The secondary endpoints were length of hospital stay, numbers of days required to resume normal outdoor activities and work, recurrence rate and incidence of chronic pain.

\section{Dosage and Application}

Fibrin glue is supplied as kit consisting of 2 separate $1 \mathrm{ml}$ vials. One vial of fibrinogen and factor x111(containing 55-85mg/ $\mathrm{ml}$ fibrinogen) and the other vial of thrombin and calcium (containing 800-1200 iu/ml of human thrombin).

The kit are stored at refrigerator ( 2 degree to 8 degree) and when used less than 24 hours, room temperature.

During the operation each vial is thawed with $2 \mathrm{ml}$ sterile water and with use of specific applicator device containing 2 syringes. By drip the content of the applicator device into the mesh each drip $0.1-0.2 \mathrm{ml}$ at different sites to produce a thin layer. At this moment after mixture of fibrinogen with thrombin lead to formation of a sealant layer of fibrin within minutes after mixture.

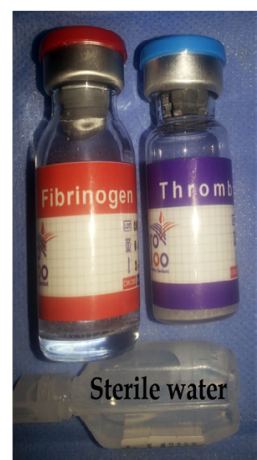

To evaluate complications resulting from the operation, followup clinical assessments took place at 7 days, one month and 6 months. This included questioning about post-operative pain. When present, pain was ranked as slight, moderate or severe. 


\section{Post-operative Pain Assessment}

We used numerical pain rating scale for postoperative pain measurement according to McLafferty and Farley [24] and patient complain and satisfaction.

\section{Histological Examination}

At the end of the Fibrin Glue Mesh Fixation, small pieces from the mesh fixed with the posterior wall of the inguinal canalconjoint tendon were taken and fixed immediately in neutral buffered formalin $10 \%$ for the histological studies. The fixed specimens were processed using the usual histological techniques; dehydrated in ascending grades of ethanol series, cleared in benzene and embedded in paraffin. 5-7 $\mu \mathrm{m}$ thick sections were prepared and mounted on glass slides. These were dewaxed in xylene, hydrated in descending grades of ethanol series and stained with Harris's hematoxylin and Eosin (H\&E) and Green Masson's Trichrome according to Bancroft and Gamble [23]. The microphotographies were taken using a digital Dsc-W 130 super steady cyper shot camera (Sony, Japan) connected to an Olympus BX 21 light microscope.

\section{Result}

We employed a fibrin glue mesh fixation in 30 patients (group A) and sutures mesh fixation using vicryl in 30 patients (group B). All the patients included in this study were male and aged between 17 and 60 years old with exclusion of recurrent, obstructed and strangulated.

There were no intra-operative complications. All patients went home $24 \mathrm{~h}$ after the operation and none required readmission to hospital. No sepsis, mesh rejection or other complications such as haematoma or seroma were recorded at 7-day follow-up. At 3 months, in group B, the only complications seen were reports of moderate pain at the pubic tubercle in three patients $(10 \%)$. After receiving of the non-steroidal anti-inflammatory, the pain was disappeared after 6 months in two of patients. Meanwhile, the third one, the pain was disappeared after 12 months. At 12-month follow-up, no recurrences or late complications, such as scar immobility/fibrosis, neuralgia or scrotal hyperesthesia, were seen.

Regarding the postoperative pain, patients of group A were returned to their normal activity from 10 days to 2 weeks after operation. Meanwhile, patients of group B take more time from 2 weeks to 6 weeks for returning to normal activity.
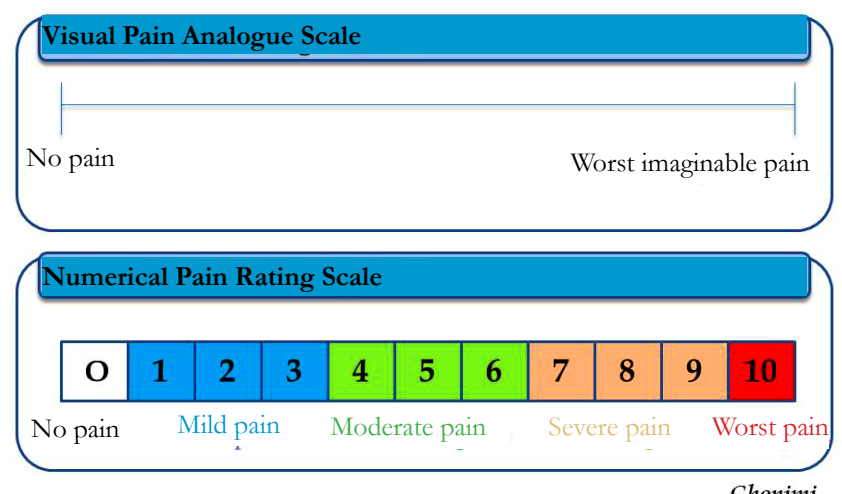

We used numerical pain rating scale for post-operative pain measurement. The patients were asked to indicate the intensity of existing less and worst pain levels over the 24 hours postoperative on a scale of 0 (No pain) to 10 (Worst imaginable pain). In group A (Fibrin glue mesh fixation), the pain was ranged from mild to moderate. Meanwhile, in group B (Suture mesh fixation), the pain was ranged from moderate to severe.

Regarding the post-operative treatment, patients of group A were needed to take 2 ampules of Diclofenac $75 \mathrm{mg}$ I/M; one after 2 hours and the second one after 12 hours post-operation and then complete to 1 st week with oral Diclofenac. This was enough to release post-operative acute pain but for releasing the postoperative chronic pain, the patients of group A were needed only oral analgesic for one week post operation.Only 6 patients were needed additional analgesic e.g. Tramadol $50 \mathrm{mg} \mathrm{I} / \mathrm{V}$ one injection in the first 12 hours post-operative.

Meanwhile, patients of group B were needed to take ampule of Diclofonac $75 \mathrm{mg} \mathrm{I} / \mathrm{M}$ each 12 hours for at least 48-72 hours post operation. 25 patients were needed additional analgesic; Tramadol $50 \mathrm{mg} \mathrm{I} / \mathrm{V}$ one injection for early 12 hours and 5 patients were needed two injections of $50 \mathrm{mg}$ IV tramadol one each 12 hours in the 24 hours post-operative. This was enough to release postoperative acute pain but for releasing the post-operative chronic pain, the 25 patients of group B were needed continues oral analgesic for at least 2 weeks and 3 patients were needed one month post operation and only two patients were needed 2 months oral analgesic.

Regarding the antibiotics protocol in the present investigation, in both two groups, just before induction of anesthesia, $1 \mathrm{gm}$ of a third generation cephalosporin; Ceftriaxone were given I/V and post-operative dose was $1 \mathrm{gm} / 24$ hours for 5 days post-operative as prophylaxis.

\section{Histological Observation}

With H\&E and Green Masson's Trichrome stains, colorless spherical structures of the posterior wall of the inguinal canalconjoint tendon were observed and some of them were appeared irregular polygonal shaped. Meanwhile, under polarized light, the brightly birefringent were observed filling these colorless structures. Furthermore, with Green Masson's Trichrome stain, massive wavy fibrin threads forming bundles were observed filling all the spaces in between the polypropylene threads of mesh fixing the later with the surrounding tissues. Moreover, numerous fibroblasts were observed in between the fibrin bundles which distributed in different directions (Figure 1 and 2).

\section{Discussion}

The important role of human fibrin glue (HFG) in surgery is supported by extensive experience acquired at an international level. A considerable body of literature has confirmed the effectiveness of this product and has also demonstrated its excellent local tolerability and lack of adverse effects and contraindications [12, 13]. Antifibrinolytic agents such as aprotinin are included in the preparation to enhance the life span of the sealant and prolong its effectiveness. In addition to its haemostatic action, the fibrinogen component gives the product its tensile strength and adhesive 
Figure 1 a,b. a). A photomicrograph of the posterior wall of the inguinal canal-conjoint tendon (T) in fibrin glue mesh fixation showing colorless spherical structures which some of them appeared irregular polygonal shaped (arrow heads) surrounded with massive fibrin threads (arrow). b)Imageofthesameslideunderthepolarizingmicroscopeshowingthe brightly birefringencethat filling the colorless spherical structures (arrow heads) and massive wavy fibrin threads forming bundles filling all the spaces in between the polypropylene threads of mesh fixing the later with the surrounding tissues (arrow).

Inset box is the higher magnification of figure $B$ showing the same.
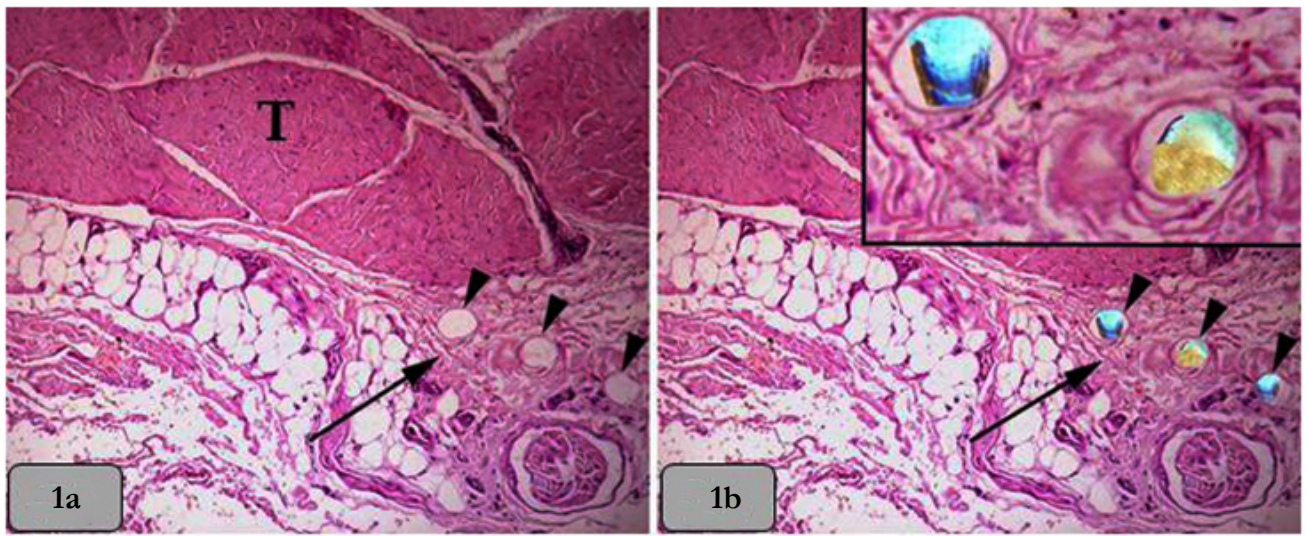

Stain: a, b) H\& E $\quad$ a, b) Obj.x10: Oc.x10. Inset box: Obj.x40: Oc.x10

Figure 2 a, b. a) A photomicrograph of the posterior wall of the inguinal canal-conjoint tendon in fibrin glue mesh fixation showing colorless spherical structures (arrow heads) surrounded with massive fibrin threads (arrow). b) showing the same structures of Figure A but under polarized light, the brightly birefringent were observed filling the colorless spherical structures (arrow heads) and massive wavy fibrin threads forming bundles filling all the spaces in between the polypropylene threads of mesh fixing the later with the surrounding tissues (arrow). Inset box is the higher magnification of figure B

showing the same and some fibroblasts were observed in between the fibrin bundles which distributed in different directions.

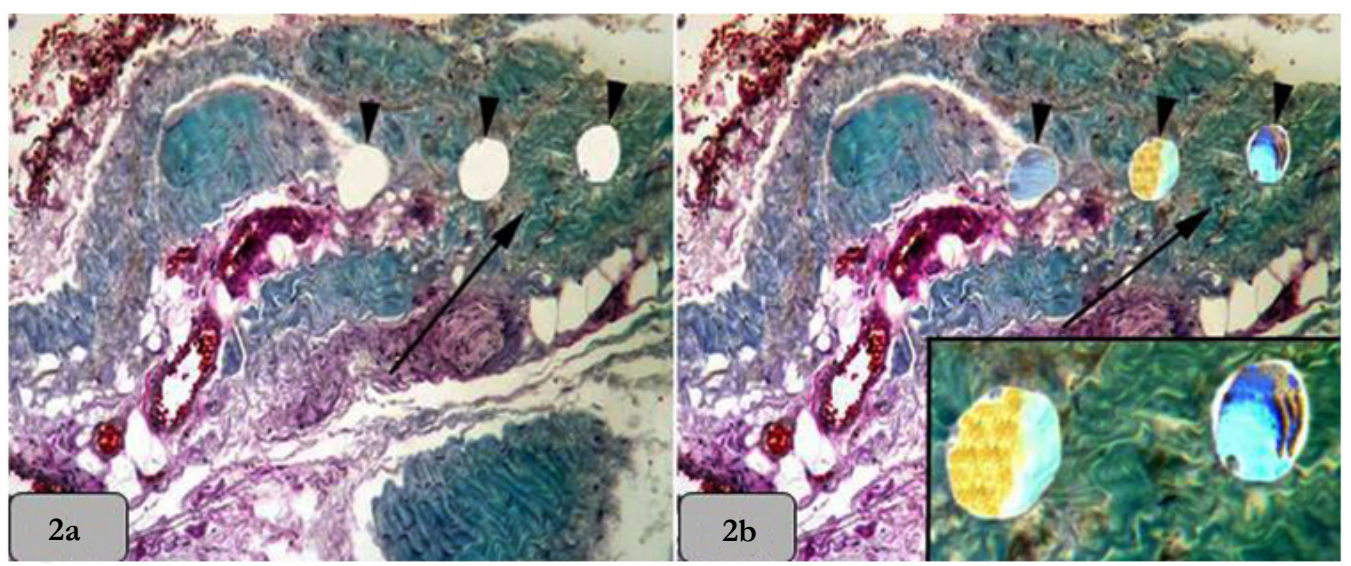

Stain: a, b) Green Masson's trichromea, b) Obj.x20: Oc.x10. Inset box: Obj.x40: Oc.x10

properties, and the thrombin component promotes fibroblast proliferation [14]. As a consequence of these properties, HFG has contributed to the improvement of surgical procedures and in some cases, the development of new techniques, such as the treatment of fistulous complications following bowel anastomosis $[12]$.

In a previous study, we found that HFG was effective in preventing local haemorrhagic complications after hernioplasty in patients with concurrent coagulation disorders [16]. Studies by other investigators have firmly established the adhesive properties of this biological sealant $[17,18]$, and excellent results with the use of HFG for prosthetic mesh fixation in laparoscopic extra peritoneal inguinal hernia repair have been reported [19].

A preliminary study on the use of N-butyl-2-cyanoacrylate as an alternative adhesive for tension-free inguinal hernia repair has shown that this sealant can clog mesh pores and promoting the septic complications [20]. More recently, a clinical trial examining the use of N-butyl-cyanoacrylate for fixing the mesh prosthesis in inguinal hernia repair has shown promising early results [21]. However, the cyanoacrylates are chemical sealants and dry too quickly (within 5-7s), forming a rigid binding. This means that the mesh can be fixed only at a few points at the edges, so that haematomas or seromas can occur under the prosthesis (Helbling and Schlumpf reported a $13.5 \%$ incidence of early haematomas [21]. Furthermore, these sealants dry with an exothermic reaction and show a degree of histotoxicity. In contrast, HFG is a biological glue that naturally coagulates and is reabsorbed without changing tissue or mesh characteristics.

Our study shows that mesh fixation with HFG is suitable for use 
in open tension-free inguinal repair. There were no complications related to the technique. In particular, no haematomas, seromas or neuralgias were observed over 12 months of follow-up. Moreover, the mean operating time was shorter when compared with the mean operating time of the classic Lichtenstein technique at our hospital (35 vs $50 \mathrm{~min}$, respectively), and at other hospitals (e.g., $60 \mathrm{~min}$ ) [15]. In addition, surgeons reported a low level of perceived difficulty and a high level of satisfaction.

In conclusion, our results confirm the efficacy of mesh fixation with HFG and support the viability of a sutureless Lichtenstein procedure. Whether this approach becomes widespread will depend on further evaluation in multicenter controlled trials.

GMF is comparable to SMF in terms of postoperative complications, postoperative pain, chronic groin pain and length of hospital stay and patient satisfaction. GMF is associated with a reduced operative time compared with SMF. Based on the results of this review the GMF approach may be considered as a better alternative for mesh fixation in OIHR [22].

Histologically, with H\&E and Green Masson's Trichrome stains, colorless spherical structures of the posterior wall of the inguinal canal-conjoint tendon were observed and some of them were appeared irregular polygonal shaped. Meanwhile, under polarized light, the brightly birefringent were observed filling these colorless structures. These findings are indicating that the polypropylene mesh; non absorbable mesh for hernia repair is un stained material where it gave negative reaction with H\&E and Green Masson's Trichrome stains.

With Green Masson's Trichrome stain, massive wavy fibrin threads forming bundles were observed filling all the spaces in between the polypropylene threads of mesh fixing the later with the surrounding tissues. This finding is clarifying that a sealant layer of fibrin is formed immediately within seconds to few minutes after mixing fibrinogen with thrombin and this investigation is in parallelism with the findings of $[7,8]$ who claimed that in practice, it is a two-component system in which a solution of concentrated fibrinogen and factor XIII are combined with a solution of thrombin and calcium to form a coagulum, simulating the final stage of the clotting cascade. Once the thrombin/calcium is combined with the fibrinogen/factor XIII, a fibrin clot forms in seconds, or somewhat slower if a more dilute form of thrombin is used.

Photograph showing the division of the external oblique apponurosismuscleto open the inguinal canal and deliver the spermatic cord.

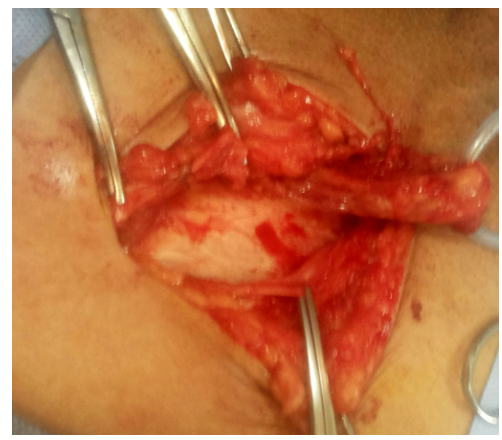

Photographs showing the meshadjustment (right figure)and then applying the mesh in the inguinal canal after transferring of excision of the hernial sac (left figure).

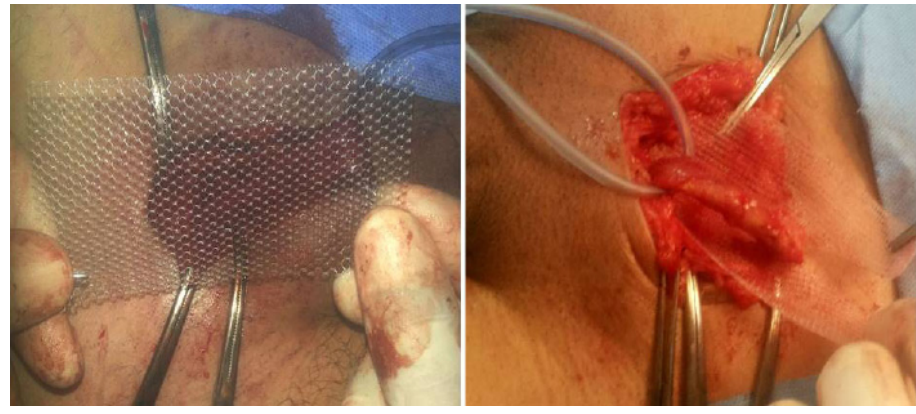

Photographs showing the applying of the fibrin glue in mesh fixation using specific applicator with double syringes ended with one open; one syringe has fibrinogen and the second one has thrombin.

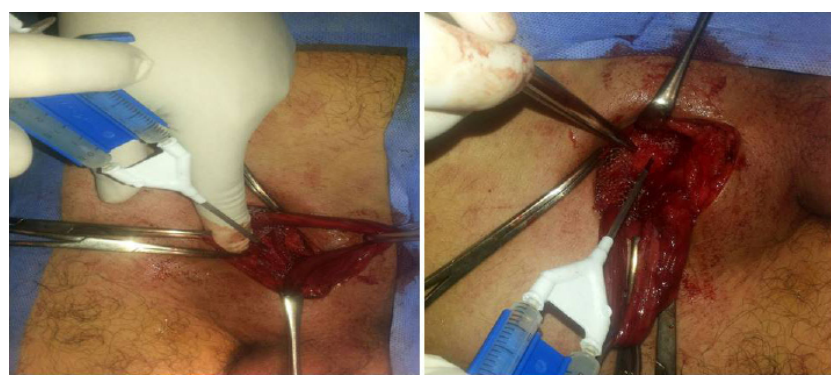


Photograph showing the finishing of the mesh fixation with fibrin glue.

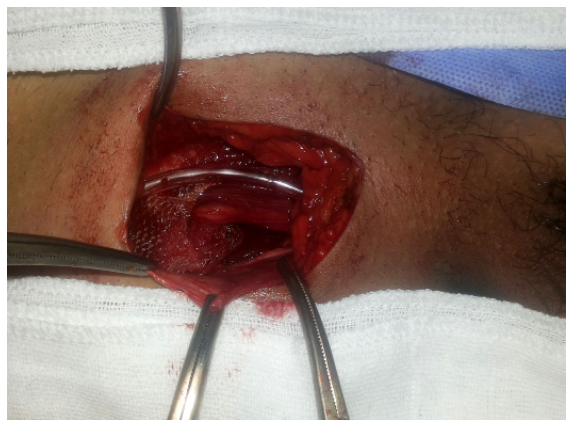

Photographs showing the sutures mesh fixation using vicryl.

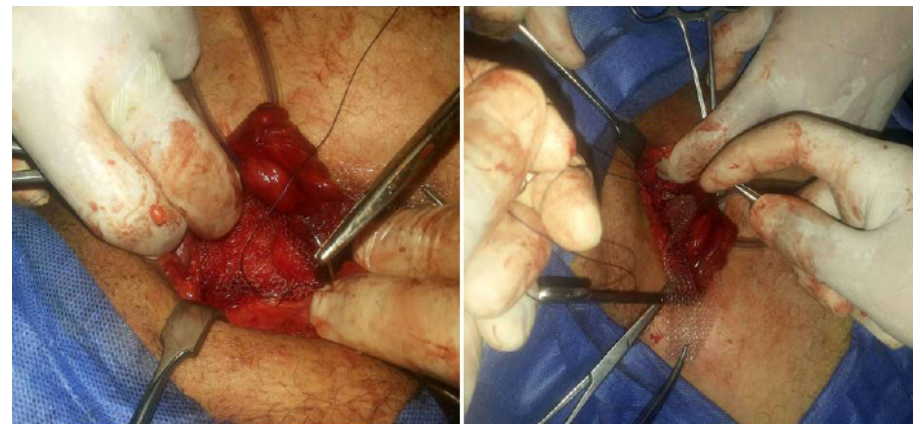

Photograph showing the closure of the wound with subcuticular suture.

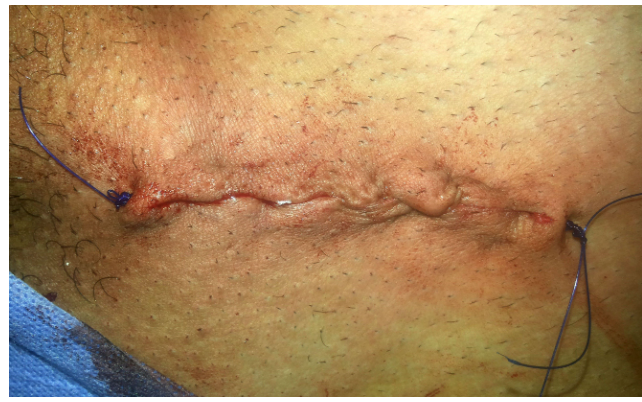

Numerous fibroblasts were observed in between the fibrin bundles which distributed in different directions. Such investigation is in agreement and supported by the finding of $[9,10]$ who clarified that the antifibrinolytic agents such as aprotinin are included in the preparation to enhance the life span of the sealant and prolong its effectiveness. In addition to its haemostatic action, the fibrinogen component gives the product its tensile strength and adhesive properties, and the thrombin component promotes fibroblast proliferation [9]. Thrombin is an enzyme and converts fibrinogen into fibrin monomers between 10 and 60 seconds giving rise to a three-dimensional gel [10].

\section{Conclusion}

The present investigation concluded that the use of fibrin glue is better than sutures in tension free inguinal hernia repair using mesh technique. There are less operative time, postoperative pain, chronic pain with early return of the patients to normal activities and work in group A using fibrin glue in mesh fixation in inguinal hernia repair.

\section{References}

[1]. Amid PK, Shulman AG, Lichtenstein IL (1994) A critical evaluation of the
Lichtenstein tension-free hernioplasty. Int Surg. 79(1): 76-79.

[2]. Amid PK, Lichtenstein IL (1998) Long-term results and current status of the Lichtenstein open tension-free hernioplasty. Hernia. 2(2): 89-94.

[3]. Kingsnorth AN, Bowley DM, Porter C (2003) A prospective study of 1000 hernias: results of the Plymouth Hernia Service. Ann R Coll Surg Engl. 85(1): $18-22$.

[4]. Bay-Nielsen M, Perkins FM, Kehlet H, Danish Hernia Database (2001) Pain and functional impairment 1-year after inguinal herniorrhaphy: a nationwide questionnaire study. Ann Surg. 233(1): 1-7.

[5]. Nienhuijs S, Kortmann B, Boerma M, Strobbe L, Rosman C (2004) Preferred mesh-based inguinal hernia repair in a teaching setting: results of a randomized study. Arch Surg. 139(10): 1097-1100.

[6]. Paajanen H (2002) Do absorbable mesh sutures cause less chronic pain than non-absorbable sutures after Lichtenstein inguinal herniorraphy? Hernia 6(1): 26-28.

[7]. Kram HB, Nathan RC, Mackabee JR, klein SR, Shoemaker WC (1988) Clinical use of nonautologous fibrin glue. Am Surg. 54(9): 570.

[8]. Harris DM, Siedentop KH, Ham KR, Sanchez B(1987) Autologous fibrin tissue adhesive biodegration and systemic effects. Laryngoscope. 97(10): 1141.

[9]. Byrne DJ, Hardy J, Wood RAB, McIntosh R, Cuschieri A (1991) Effect of fibrin glue on the mechanical properties of healing wounds. Br J Surg. 78(7): 841-843

[10]. Spotnitz WD (2010) "Fibrin Sealant: Past, Present, and Future: A Brief Review". World J surgery. 34 (4): 632-634. doi:10.1007/s00268-009-0252-7. PMID 19820991.

[11]. Alving BM, Weinstein MJ, Finlayson JS, Menitove JE, Fratantonic JC (1995) Fibrin sealant: summary of a conference on characteristics and clinical uses. Transfusion. 35(9): 783.

[12]. Canonico S (2003) The use of human fibrin glue in the surgical operations. Acta Biomed. 74(2): 21-25. 
[13]. Houston KA, Rotstein OD (1988) Fibrin sealant in high-risk colonic anastomoses. Arch Surg. 123(2): 230-234.

[14]. Byrne DJ, Hardy J, Wood RAB, McIntosh R, Cuschieri A (1991) Effect of fibrin glue on the mechanical properties of healing wounds. $\mathrm{Br} \mathrm{J}$ Surg. 78(7): 841-843.

[15]. Nienhuijs S, Kortmann B, Boerma M, Strobbe L, Rosman C (2004) Preferred mesh-based inguinal hernia repair in a teaching setting: results of a randomized study. Arch Surg. 139(10): 1097-1100.

[16]. Canonico S, Sciaudone G, Pacifico F, Santoriello A (1999) Inguinal hernia repair in patients with coagulation problems: prevention of postoperative bleeding with human fibrin glue. Surgery. 125(3): 315-317.

[17]. Chevrel JP, Flament JB (1990) Methods thérapeutiques. Les eventrations de la Paroi Abdominale. Editorial Masson, Paris. 124-126.

[18]. Chevrel JP, Rath AM (1997) The use of fibrin glues in the surgical treatment of incisional hernias. Hernia. 1(1): 9-14.
[19]. Katkouda N, Mavor E, Friedlander MH, Mason RJ, Kiyabu M, et al., (2001) Use of fibrin sealant for prosthetic mesh fixation in laparoscopic extraperitoneal inguinal hernia repair. Ann Surg 233:18-25.

[20]. Farouk R, Drew PJ, Qureshi A, Roberts AC, Duthie GS, et al., (1996) Preliminary experience with butyl-2-cyanoacrylate adhesive in tension-free inguinal hernia repair. Br J Surg. 83(8): 1100.

[21]. Helbling C, Schlumpf R (2003) Sutureless Lichtenstein: first results of a prospective randomised clinical trial. Hernia. 7(2): 80-84.

[22]. N Ladwa, MS Sajid, P Sains, MK Baig (2013) Suture mesh fixation versus glue mesh fixation in open inguinal hernia repair: A systematic review and meta-analysis. Int j surg. 11(2): 128-135.

[23]. Bancroft JD, Gamble M (2001) Theory and practice of histological techniques. (5th Edn)., Churchill livingstone. 281-285.

[24]. McLafferty E, Farley A (2008) Assessing pain in patients. Nursing Standard. 22(25): 42-46. 